\title{
An application of an edge effect based vacuum blower to a lyophilizer
}

\author{
M. Kitamura, M. Tsutahara \& H. Sasaki \\ Graduate School of Science and Technology, Kobe University, Japan
}

\begin{abstract}
In the rarefied gas, the edge effect flow is induced at a heated edge of a flat plate and its mechanism is well known. Usually edge effect flow is generated at both sides of the flat plate and the effects cancel each other out because the directions of the flows are completely opposite. By rounding the edge of a flat plate at one side we can obtain one-way flow and can use this flow for a vacuum blower. By confirming the characteristic of the effect of this edge effect flow we try to use this flow as an edge effect blower for lyophilizers. We confirmed that there is a pressure difference between the inlet and outlet of this blower caused by this edge effect flow and also this pressure difference is very obvious in the 200-400 $\mathrm{Pa}$ range of the surrounding pressure. We set this edge effect blower in a vacuum tank and examined whether this blower could promote the sublimation phenomenon of water. We confirmed that the sublimation was increased by using this blower. We also performed a numerical analysis using the finite difference lattice Boltzmann method to simulate the sublimation phenomena. Gas flows caused by evaporation and condensation phases are simulated.

Keywords: rarefied gas, edge effect, lattice Boltzmann method, finite difference method, evaporation, condensation.
\end{abstract}

\section{Introduction}

We can usually consider the gas to be a continuum and describe a phenomenon by applying the Navier-Stokes equations as the governing equations. However, for low pressure gases, the Navier-Stokes equations and the no-slip boundary condition cannot be applied, because the mean free path of molecules cannot be neglected. We can describe such a gas phenomenon by the Boltzman equation. 
In the rarefied gas, the thermal transpiration flow is induced when a solid wall has a temperature gradient [1-3]. The edge effect flow is also induced at the edge of a flat plate and its mechanism is the same as thermal transpiration flow.

As an engineering application of this edge effect flow, we take up the lyophilizer this time. There is no system of the ventilation effect in the current lyophilizers that is necessary for the sublimation process.

By applying an edge effect flow for the ventilation of the lyophilizer the rise of water sublimation speed is examined by experiments. The effectiveness of numerical simulation by the FDLBM is also checked by comparing it with the results by a molecular gas dynamics based calculation.

\section{Experiments}

We made an edge effect based vacuum blower (edge effect blower) using the edge effect flow. The edge effect flow usually occurs at both edges of a plate and it is offsetting. But by rounding one edge we can obtain a flow only for one direction [4]. We confirmed the pressure at the outlet of the blower was raised. We also confirmed that this blower is effective for speeding up the process of 1 sublimation.

\subsection{Experiments by edge effect blower}

\subsubsection{Experimental device and method}

We use the accumulation unit that incorporated edge plates in that unit. A schematic view of the experimental device is shown in Fig 1. The accumulation unit of edge effect blower is settled in a vacuum chamber.

The pressure in the chamber and the accumulator are measured by Pirani gauges. Each edge plate is equipped with heaters to be heated.

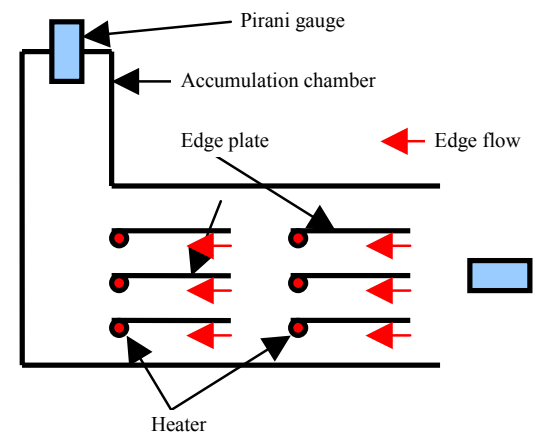

Figure 1: Schematic view of the edge effect blower. 
The value of the vacuum gauge which is installed in a vacuum tank shows a back pressure of an edge effect blower, and a difference between a value in the edge effect blower and that of the vacuum tank is understood as a rise of pressure by an edge effect flow. After confirming at arrival the pressure in a vacuum tank at about $90 \mathrm{~Pa}$, the pressure measurement is started.

\subsubsection{Test results and discussion}

The test results are shown in Fig. 2. The measurements are done in two cases. One case (case 1) is tested without heating the edge plate dotted square marks. The second case (case 2 ) is measured with heating the edge plate dotted diamond marks.

We can confirm that the pressure rises in case 2 by heating an edge plate in the back pressure of 200-400 Pa region. It is thought that the edge effect is most effective in this region. Drops of pressure in case 2 around $400 \mathrm{~Pa}$ is caused by the characteristics of gauges.

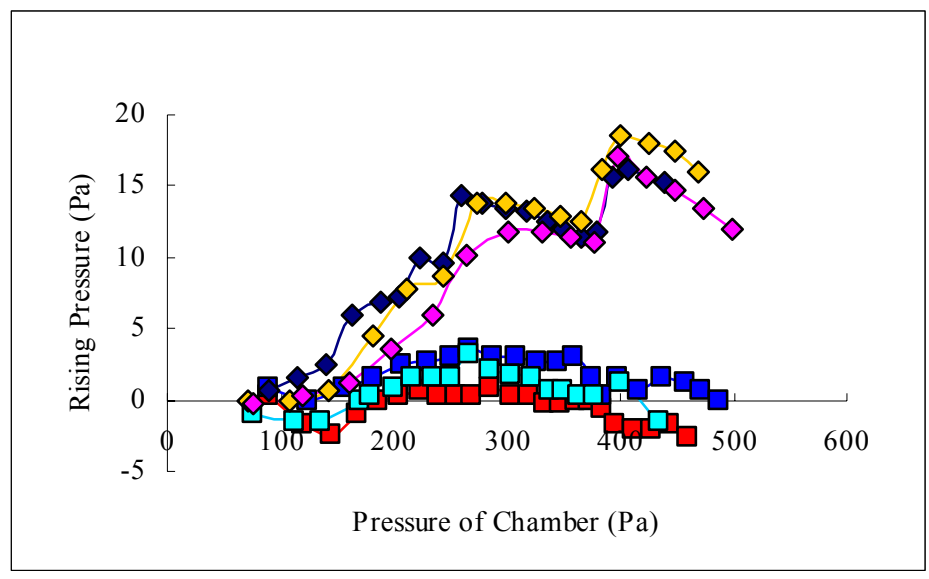

Figure 2: Relation between pressure rise and back pressure in vacuum chamber.

\subsection{An application of edge effect blower to a lyophilizer}

An edge effect blower is installed in a vacuum tank and the speeding up the process of the sublimation is examined.

\subsubsection{Experimental device and method}

A view of an edge effect blower is shown in Fig 3. This blower consists of three plates bent at one end comprising heater in one row, and has two rows of plates. The experimental apparatus is shown in Fig. 4.

The performance of an edge effect blower is tested by the sponge soaked with water and frozen up. The difference of quantity of water left in the sponge is measured by the following three cases. 


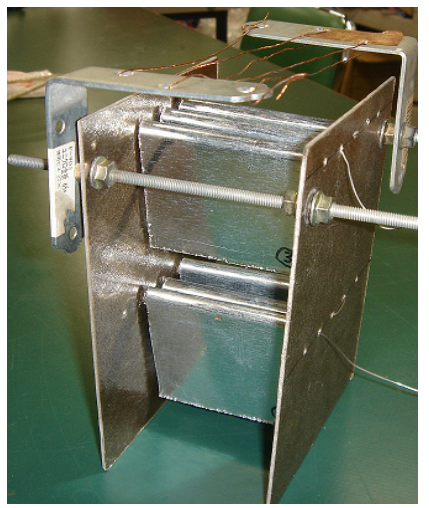

Figure 3: $\quad$ Edge effect blower.

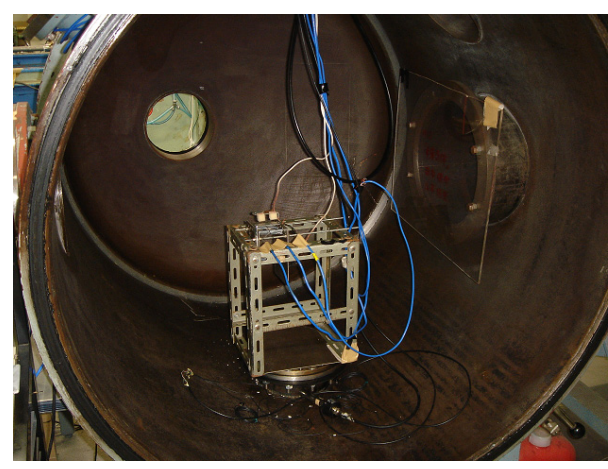

Figure 4: View of experimental apparatus.

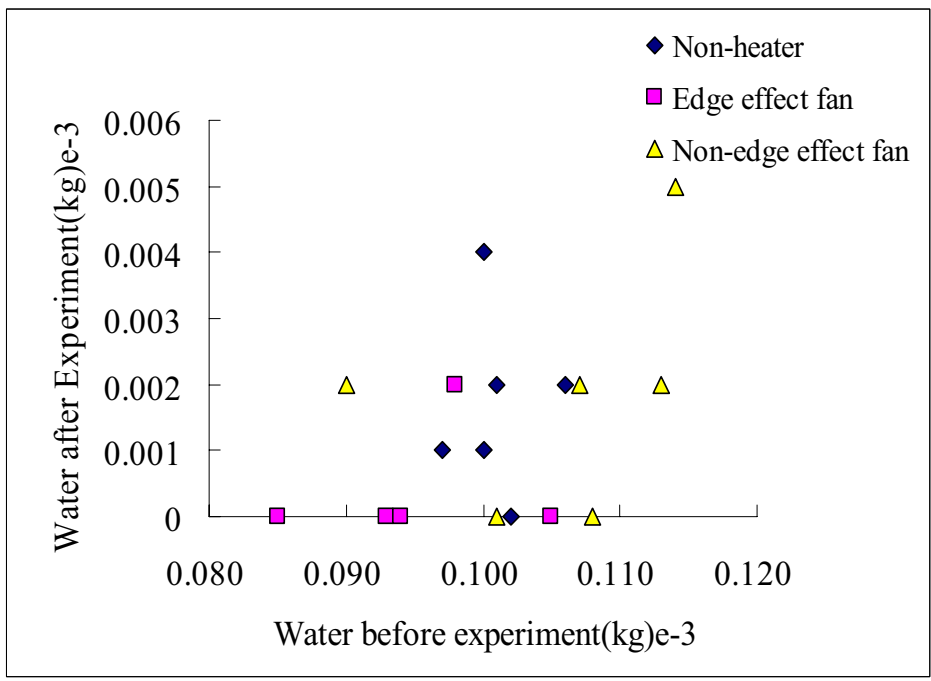

Figure 5: Relation between contained water before and after experiment.

Case 1: Experiment with edge effect plate of no heat.

Case 2: Experiment with edge effect plate of heat

Case 3: Experiment without edge effect plate

\subsubsection{Test results and discussion}

The test result is shown in Fig. 5 in the case of a start pressure $200 \mathrm{~Pa}$ in the vacuum chamber. It takes about 4 minutes before arriving at the experimental start pressure $200 \mathrm{~Pa}$. During the experiment the pressure rises about $50 \mathrm{~Pa}$ in the 
vacuum chamber. Some dispersion of the data is seen, but, as for the case without the source of heat, the quantity of water sublimation decreases as the pressure rises.

Cases 1 and 3: The test results of case 3 (without edge effect plate), as the pressure rises, the quantity of water sublimation decreases as well as case 1 (without the heat). This cause is similar to the case without the source of heat.

Case 2: In the case of the edge effect blower, quantity of water sublimation becomes greatest at the starting pressure $200 \mathrm{~Pa}$ in the vacuum chamber. Therefore, it seems that sublimation is promoted by the ventilation effect of the edge effect blower. Although the dispersion is rather large, it seems that a ventilation effect by the edge effect flow is effective on lyophilization in the range of $100-400 \mathrm{~Pa}$.

\section{Numerical analysis}

The lattice Boltzmann method (LBM) is the computational tool to analyse the continuous thermal viscous fluid. The flow in the vacuum chamber is governed by the Navier-Stokes equations except the Knudsen layer near the sublimation surface. The LBM model is a strongly discretized version of the Boltzmann equation. Therefore the LBM can be considered to simulate the flow except the Knudsen layer without any special treatment for the boundary conditions. In this paper, the finite difference lattice Boltzmann method (FDLBM) is used. The two-dimensional thermal model (the D2Q21model) $[5,6]$ of FDLBM is presented briefly in section 3.1. The numerical analysis method is presented in section 3.2.

\subsection{Finite difference lattice Boltzmann method}

The discrete BGK equation for the FDLBM is written as follows with the distribution function $f_{i}^{k}(\mathbf{x}, t)$ having the particle velocity $c_{i}$

$$
\frac{\partial f_{i}}{\partial t}+\mathbf{c}_{i} \frac{\partial f_{i}}{\partial \mathbf{x}}=-\frac{1}{\phi}\left[f_{i}-f_{i}^{(0)}\right]
$$

where $\phi$ is the collision parameter (the relaxation time) and $f_{i}^{0}$ is the local equilibrium distribution function chosen to satisfy the Navier-Stokes equation. The local equilibrium distribution function is defined as follows in the case of the thermal model.

$$
\begin{array}{r}
f_{i}^{0}=\omega_{i} \rho\left(1-2 B u_{\alpha} c_{i, \alpha}+2 B^{2}\left(u_{\alpha} c_{i, \alpha}\right)^{2}+B u^{2}\right. \\
\left.-\frac{4}{3} B^{3}\left(u_{\alpha} c_{i, \alpha}\right)^{3}-2 B^{2} u_{\alpha} c_{i, \alpha} u^{2}\right)
\end{array}
$$

The macroscopic variables for continuous fluids are defined as

$$
\rho=\sum_{i} f_{i}=\sum_{i} f_{i}^{(0)}
$$




$$
\begin{gathered}
\rho \mathbf{u}=\sum_{i} f_{i} \mathbf{c}_{i}=\sum_{i} f_{i}^{(0)} \mathbf{c}_{i} \\
\frac{1}{2} \rho u^{2}+\rho e=\sum_{i} \frac{1}{2} f_{i} c_{i}{ }^{2}=\sum_{i} \frac{1}{2} f_{i}^{(0)} c_{i}^{2}
\end{gathered}
$$

for the density, the momentum and energy, respectively, where e is the internal energy per unit mass.

For the finite difference lattice Boltzmann method, the governing differential equation is discretized in finite difference schemes, and the following calculation procedures are employed. The time integration is performed by the second-order Runge-Kutta method and the third-order upwind scheme is employed for space differential.

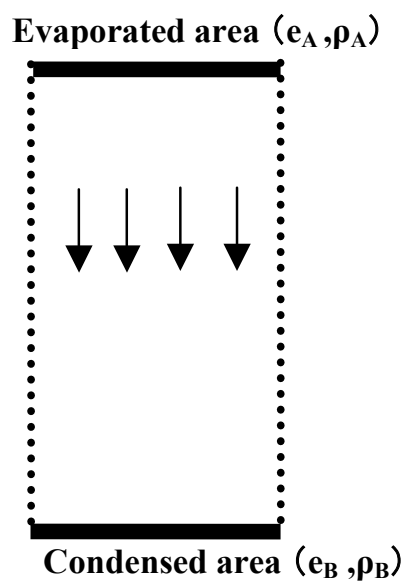

(a) Parallel condensed phases

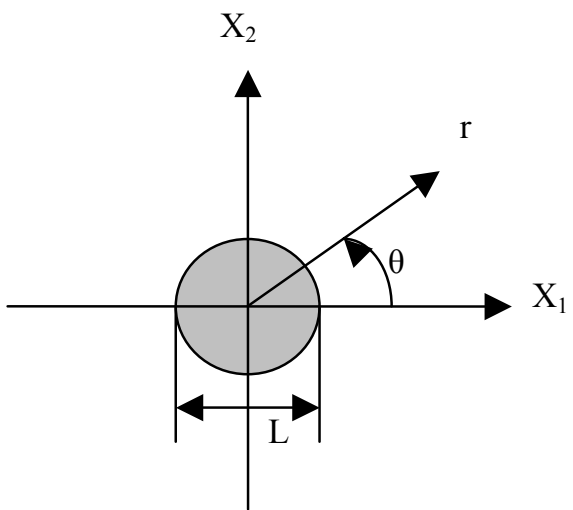

(b) Cylindrical condensed phase

Figure 6: Schematic diagram.

\subsection{Method of analysis}

\subsubsection{Evaporation and condensation between two parallel plates}

One-dimensional behaviours of gas between two parallel condensed phases is considered as shown in Fig. 6(a). Both condensed phases spread infinitely and are kept at different temperatures. We only consider the condensed gas. Evaporation occurs at the high temperature condensed phase and condensation in the low temperature condensed phase and flow of the gas occurs towards the low temperature side from the high temperature side.

It is assumed that the temperature is $T_{A}$ (internal energy $e_{A}$ ) at the high temperature side and $T_{B}$ (internal energy $e_{B}$ ) at the low temperature side, and the saturated vapour pressure of gas at each side is given $p_{A}$ and $p_{B}$. (Fig. 6(a)) The local equilibrium distribution functions defined by these temperatures and the pressures (densities), and the zero flow velocity are used. We compare the flows in the steady state to those given by the molecular dynamics based calculations. 


\subsubsection{Evaporation from cylindrical condensed phase}

Two-dimensional evaporation from a cylindrical surface in an infinitely expanded domain as shown in Fig. 6(b) is also considered. We assume the temperature of the cylinder surface is $T_{w}$ (internal energy e) and assume saturated vapour pressure of gas is $p_{w}$. The temperature and the pressure at infinity are $T_{\infty}$ and $p_{\infty}$, respectively. The local equilibrium distribution function defined by the temperature, the pressure ( density) and the zero flow velocity is used.

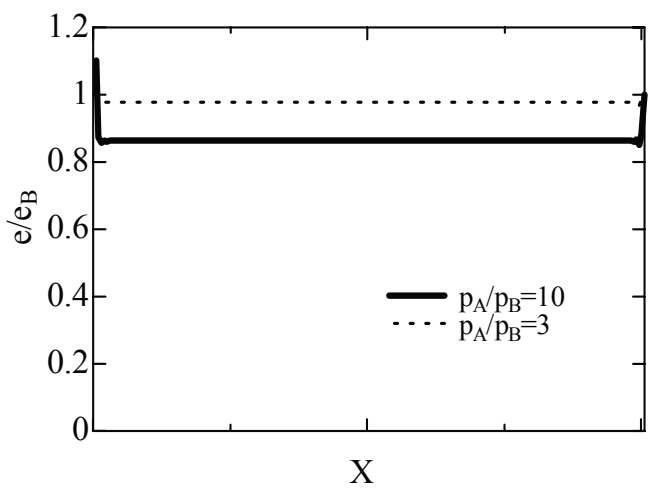

(a) $T / T_{B}$

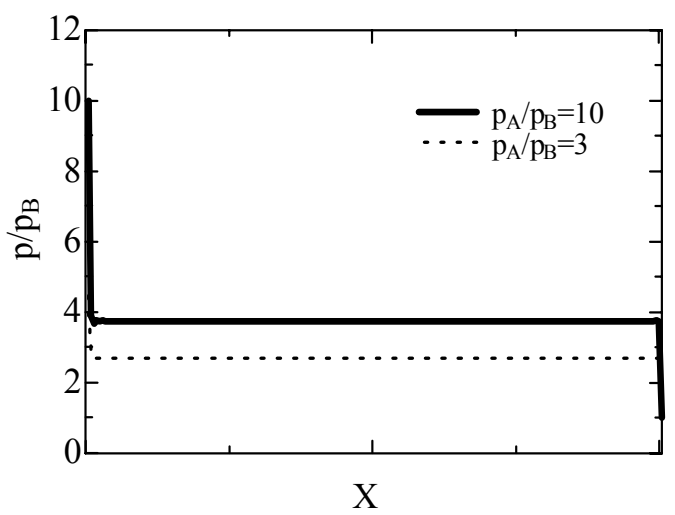

(b) $\mathrm{p} / \mathrm{p}_{\mathrm{B}}$

Figure 7: Temperature (internal energy) and pressure distributions in case of $\mathrm{p}_{\mathrm{A}} / \mathrm{p}_{\mathrm{B}}=10, \mathrm{~T}_{\mathrm{A}} / \mathrm{T}_{\mathrm{B}}=1.1$ and $\mathrm{p}_{\mathrm{A}} / \mathrm{p}_{\mathrm{B}}=3.0, \mathrm{~T}_{\mathrm{A}} / \mathrm{T}_{\mathrm{B}}=1.1$. Left side (A side) is the evaporation phase and right side ( $\mathrm{B}$ side) is the condensation phase. (a) $e / e_{B}\left(T / T_{B}\right)$ : Temperature distribution as a function of $X$, distance from $A$ for $\mathrm{p}_{\mathrm{A}} / \mathrm{p}_{\mathrm{B}}=10$ and $\mathrm{p}_{\mathrm{A}} / \mathrm{p}_{\mathrm{B}}=3$. (b) $\mathrm{p} / \mathrm{p}_{\mathrm{B}}$ : Pressure distribution as a function of $X$ for $\mathrm{p}_{\mathrm{A}} / \mathrm{p}_{\mathrm{B}}=10$ and $\mathrm{p}_{\mathrm{A}} / \mathrm{p}_{\mathrm{B}}=3$. 


\subsection{Results and discussion}

\subsubsection{Evaporation and condensation between two parallel plates}

The distributions of the temperature and the saturated pressure between both phases in case of $\mathrm{p}_{\mathrm{A}} / \mathrm{p}_{\mathrm{B}}=3.0, \mathrm{~T}_{\mathrm{A}} / \mathrm{T}_{\mathrm{B}}=1.1$, and $\mathrm{p}_{\mathrm{A}} / \mathrm{p}_{\mathrm{B}}=10.0, \mathrm{~T}_{\mathrm{A}} / \mathrm{T}_{\mathrm{B}}=1.1$ are shown in Fig. 7. The rapid changes of the pressure and the temperature in both cases arise near the condensation and the evaporation phases, but except for these layers the pressure and temperature are uniform. This shows the same tendency with the molecular gas dynamics result [7] based on the BGK equation with $\mathrm{Kn}_{\mathrm{B}}=0$.

Comparing the results for $\mathrm{p}_{\mathrm{A}} / \mathrm{p}_{\mathrm{B}}=10$ with those for $\mathrm{p}_{\mathrm{A}} / \mathrm{p}_{\mathrm{B}}=3$, a temperature descent of the gas is more remarkable in the case of $\mathrm{p}_{\mathrm{A}} / \mathrm{p}_{\mathrm{B}}=10$ because the flow from the condensed side to the evaporated side becomes fast caused by the large difference of saturated vapour pressures. The reverse temperature incline phenomenon that appeared in the results of molecular gas dynamics is not detected.

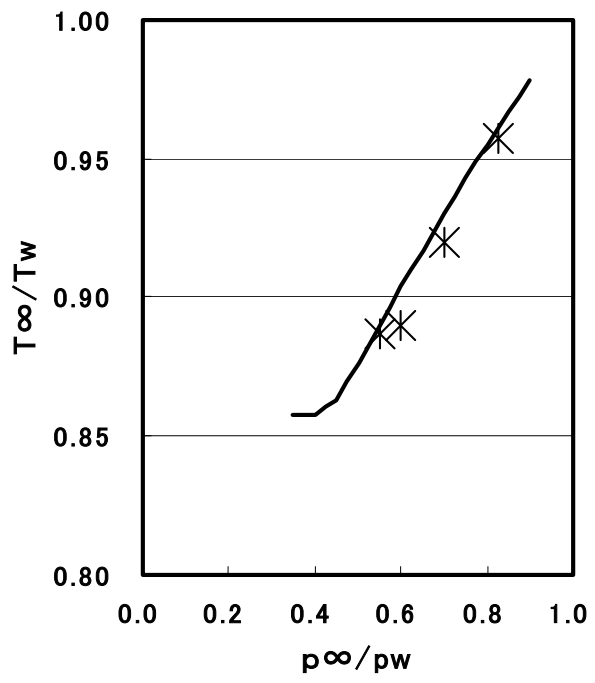

Figure 8: The temperature ratio versus the pressure ratio.

\subsubsection{Evaporation from cylindrical condensed phase}

The relation of $\mathrm{T}_{\infty} / \mathrm{T}_{\mathrm{w}}$ vs. $\mathrm{p}_{\infty} / \mathrm{p}_{\mathrm{w}}$ is shown in Fig. 8. The $\mathrm{x}$ marks show calculation results and the line shows the results by molecule gas dynamics. The calculation results by FDLBM become closer to the latter when $\mathrm{p}_{\infty} / \mathrm{p}_{\mathrm{w}}$ approaches to unity.

The pressure distributions $\mathrm{p} / \mathrm{p}_{\mathrm{w}}$ for $\mathrm{p}_{\infty} / \mathrm{p}_{\mathrm{w}}(0.82,0.70,0.61$ and 0.55$)$ are shown in figure 9 . When the pressure ratio $\mathrm{p}_{\infty} / \mathrm{p}_{\mathrm{w}}$ becomes smaller, the pressure profile $\mathrm{p} / \mathrm{p}_{\mathrm{w}}$ near the cylinder surface becomes inclined and to be smaller. These results show the same tendency as those of the molecule gas dynamic results near $\mathrm{Kn}_{\mathrm{B}}=0$ [8]. 


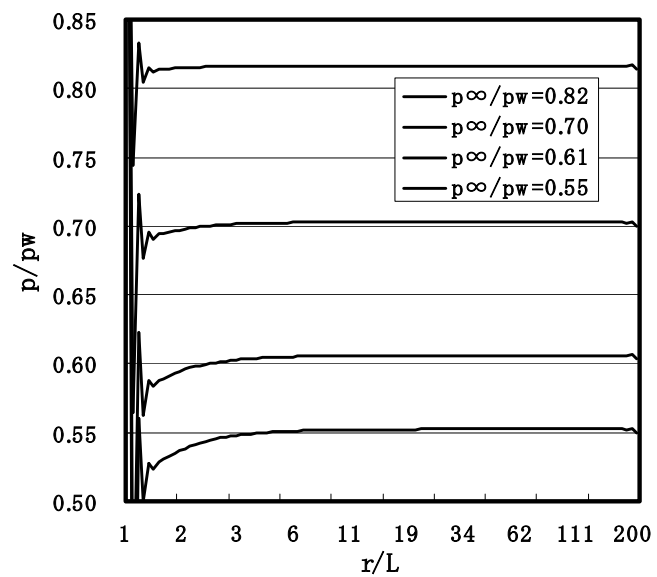

Figure 9: Distribution of non-dimensional pressure from the cylinder surface.

\section{Conclusions}

The experiment of applying an edge effect blower to lyophilization confirms that the sublimation is promoted by the presence of the edge effect blower in the pressure area of $200 \sim 400 \mathrm{~Pa}$. However, in higher vacuum domain of $1 \sim 10 \mathrm{~Pa}$, the remarkable influence is not confirmed. We need more detailed experimental work around these pressure regions and have to confirm the usefulness of the edge effect blower to a lyophilizer.

By the numerical analysis, comparison with a calculation result based on molecular gas dynamics gives a good agreement. It is confirmed that the FDLBM model (the D2Q21 model) for compressible fluid with boundary condition given by the local equilibrium distribution function is very useful for simulation of flows caused by the condensations and/or evaporations. This method is also powerful for complex shaped boundaries.

\section{References}

[1] Y. Sone, K Aoki, Molecular Gas Dynamics, Asakura-syoten 1994,in Japanese

[2] Y. Sone, Y. Waniguchi, K. Aoki, One-way flow of rarefied gas induced in a channel with a periodic temperature distribution, Phys.Fluids, 8,pp22272235, 1996

[3] Y Sone, Kinetic Theory and Fluid Dynamics, BIRKHAUSER 2002

[4] M Tutahara, K Ogawa, T Kataoka, M Shoji, Y Sakai and M Kirimuro, Study on engineering application of the Knudsen pump in rarefied gas, Transactions of the Japan Society of Mechanical Engineers NO.044-1, 2004, in Japanese. 
[5] M Tsutahara, N Takada and T Kataoka, Lattice gas and lattice Boltzmann methods, Corona-sha 1999; in Japanese.

[6] M. Tsutahara, T. Kataoka, K. Shikata, N. Takada, New Model and Scheme for Compressible Fluids of the Finite Difference Lattice Boltzmann Method and Direct Simulations of Aerodynamic Sound" Computers and Fluids, (to be published)

[7] Kazuo Aoki and Noboru Masukawa "Gas flows caused by evaporation and condensation on two parallel condensed phases and the negative temperature gradient: Numerical analysis by using a nonlinear kinetic equation" Phys. Fluids 6(3), p1379-1395 March 1994

[8] Hiroshi Sugimoto, Yoshio Sone "Numerical analysis of steady flows of a gas evaporating from its cylindrical condensed phase on the basis of kinetic theory" Phys. Fluids A 4(2), p419-440 Feb.1992 